\title{
Elucidating the Chemical Dynamics of the Elementary Reactions of the 1-Propynyl Radical $\left(\mathrm{CH}_{3} \mathrm{CC} ; \mathrm{X}^{2} \mathrm{~A}_{1}\right)$ with Methylacetylene $\left(\mathrm{H}_{3} \mathrm{CCCH} ; \mathrm{X}^{1} \mathrm{~A}_{1}\right)$ and Allene $\left(\mathrm{H}_{2} \mathrm{CCCH}_{2} ; \mathrm{X}^{1} \mathrm{~A}_{1}\right)$
}

\author{
Chao He, ${ }^{\mathrm{a}}$ Long Zhao, ${ }^{\mathrm{a}}$ Aaron M. Thomas, ${ }^{\mathrm{a}}$ Alexander N. Morozov, ${ }^{\mathrm{b}}$ Alexander M. Mebel,,${ }^{\mathrm{b}} *$ \\ Ralf I. Kaiser, a,* \\ ${ }^{a}$ Department of Chemistry, University of Hawai'i at Manoa, Honolulu, Hawaii 96822, USA \\ ${ }^{b}$ Department of Chemistry and Biochemistry, Florida International University, Miami, Florida 33199, USA
}

\section{Supporting Information}

Optimized Cartesian coordinates and vibrational frequencies for all intermediates, transition states, reactants and products involved in the reactions of the propynyl radical with $\mathrm{C}_{3} \mathrm{H}_{4}$ isomers methylacetylene and allene. The data are given in the format of an input file for the MESS package.

\footnotetext{
* Corresponding Authors:

Prof. Ralf I. Kaiser: ralfk@ hawaii.edu

Prof. Alexander M. Mebel: mebela@ @iu.edu
} 


$\begin{array}{llll}\begin{array}{l}\text { Well i1 } \\ \text { Species } \\ \text { RRHO }\end{array} & & & \\ \text { Geometry[angstrom] } & 13 & & \\ \text { C } & & & \\ \text { C } & 1.878499143663 & 0.074320087220 & -0.010846664302 \\ \text { C } & 0.696827601612 & 0.316555020683 & -0.029020431809 \\ \text { C } & 3.301037729715 & -0.237961370886 & 0.013259181454 \\ \text { C } & -0.684425450577 & 0.660458600370 & -0.056379556163 \\ \text { C } & -1.696053305814 & -0.184193036577 & 0.033154555633 \\ \text { H } & -3.162143547967 & -0.152325248443 & 0.037323999303 \\ \text { H } & 3.904240649576 & 0.653756523986 & -0.181515851973 \\ \text { H } & 3.554336825321 & -0.984945508498 & -0.745449153612 \\ \text { H } & 3.601702205406 & -0.638125762626 & 0.986659880389 \\ \text { H } & -0.911902822882 & 1.729462671664 & -0.162086863646 \\ \text { H } & -3.576432272136 & -0.739820781698 & -0.788654936126 \\ \text { H } & -3.532698580222 & 0.879650860293 & -0.063576467307 \\ \text { Core RigidRotor } & -3.567660175695 & -0.565013055486 & 0.966984308081\end{array}$

Core RigidRotor

SymmetryFactor 1.0

End

Frequencies[1/cm] 33

$\begin{array}{lll}35.7730 & 97.0023 & 121.9173 \\ 191.7035 & 248.9584 & 251.9076 \\ 353.1394 & 423.7221 & 493.1980 \\ 779.4265 & 788.9218 & 940.9038 \\ 1041.9837 & 1050.2681 & 1050.9349 \\ 1057.2879 & 1196.8857 & 1293.1013 \\ 1395.7622 & 1416.4735 & 1450.6500 \\ 1472.4331 & 1478.5188 & 1479.9298 \\ 1727.4004 & 2337.2804 & 2962.8300 \\ 2996.6655 & 3017.7072 & 3044.4150 \\ 3066.0778 & 3071.3489 & 3076.5515\end{array}$

ZeroEnergy[kcal $/ \mathrm{mol}]-60.1$

ElectronicLevels[1/cm] 1

02

End

End

!------------------------------------

!------c6h7_i2------------

Species

$\mathrm{RRHO}$

Geometry[angstrom] 13

C

$\begin{array}{lll}0.023171620331 & 0.000168588094 & 0.016676507866\end{array}$ 


$\begin{array}{lccc}\text { C } & 0.003368154470 & -0.006717807747 & 1.473474874582 \\ \text { C } & -0.014087631878 & -0.012438214196 & 2.680607794569 \\ \text { C } & -0.053937479750 & -0.019344903535 & 4.109066847061 \\ \text { C } & 1.021320538016 & -0.015476739701 & 4.879540319046 \\ \text { C } & 2.483507843895 & -0.004814714313 & 4.765324153608 \\ \text { H } & -0.992384863217 & -0.003949495308 & -0.389686583153 \\ \text { H } & 0.543111650683 & -0.878601862153 & -0.377059929053 \\ \text { H } & 0.532661523767 & 0.888646371920 & -0.368845797715 \\ \text { H } & -1.041170937486 & -0.028323588091 & 4.570693574620 \\ \text { H } & 2.913049901392 & 0.878497338869 & 5.249123905794 \\ \text { H } & 2.788972986049 & 0.002170348165 & 3.708897974919 \\ \text { H } & 2.925290693550 & -0.886502322022 & 5.240995357911\end{array}$

Core RigidRotor

SymmetryFactor 1.0

End

Frequencies[1/cm] 33

$\begin{array}{lll}8.4520 & 88.4468 & 104.6886 \\ 184.4378 & 226.3479 & 334.3562 \\ 373.2888 & 438.9542 & 568.7975 \\ 765.9380 & 806.0515 & 919.6624 \\ 1041.2495 & 1048.8050 & 1050.9366 \\ 1054.4730 & 1182.1608 & 1305.0697 \\ 1389.9509 & 1416.1599 & 1454.6234 \\ 1466.3820 & 1478.4592 & 1479.5816 \\ 1710.1607 & 2326.1343 & 2976.7631 \\ 3018.9900 & 3049.2868 & 3068.1205 \\ 3072.3753 & 3078.4952 & 3095.8441\end{array}$

ZeroEnergy $[\mathrm{kcal} / \mathrm{mol}]-60.4$

ElectronicLevels $[1 / \mathrm{cm}] \quad 1$

02

End

End

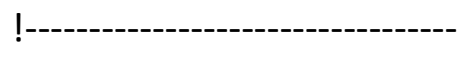

!-----c6h7_i3----------

Well i3

Species

RRHO

Geometry[angstrom] 13

C

C

$-1.277718118822$

0.045903691276

0.106852857632

$-0.072370423869$

0.068814920642

0.065014990407

C

$-2.733805105716$

0.023603819728

0.130340307141

C

1.358986762243

0.083016317514

0.080763280134

C

2.060262242515

0.309467182135

$-1.015770185569$

C

2.027906800906

$-0.176489429165$

1.427061264601 


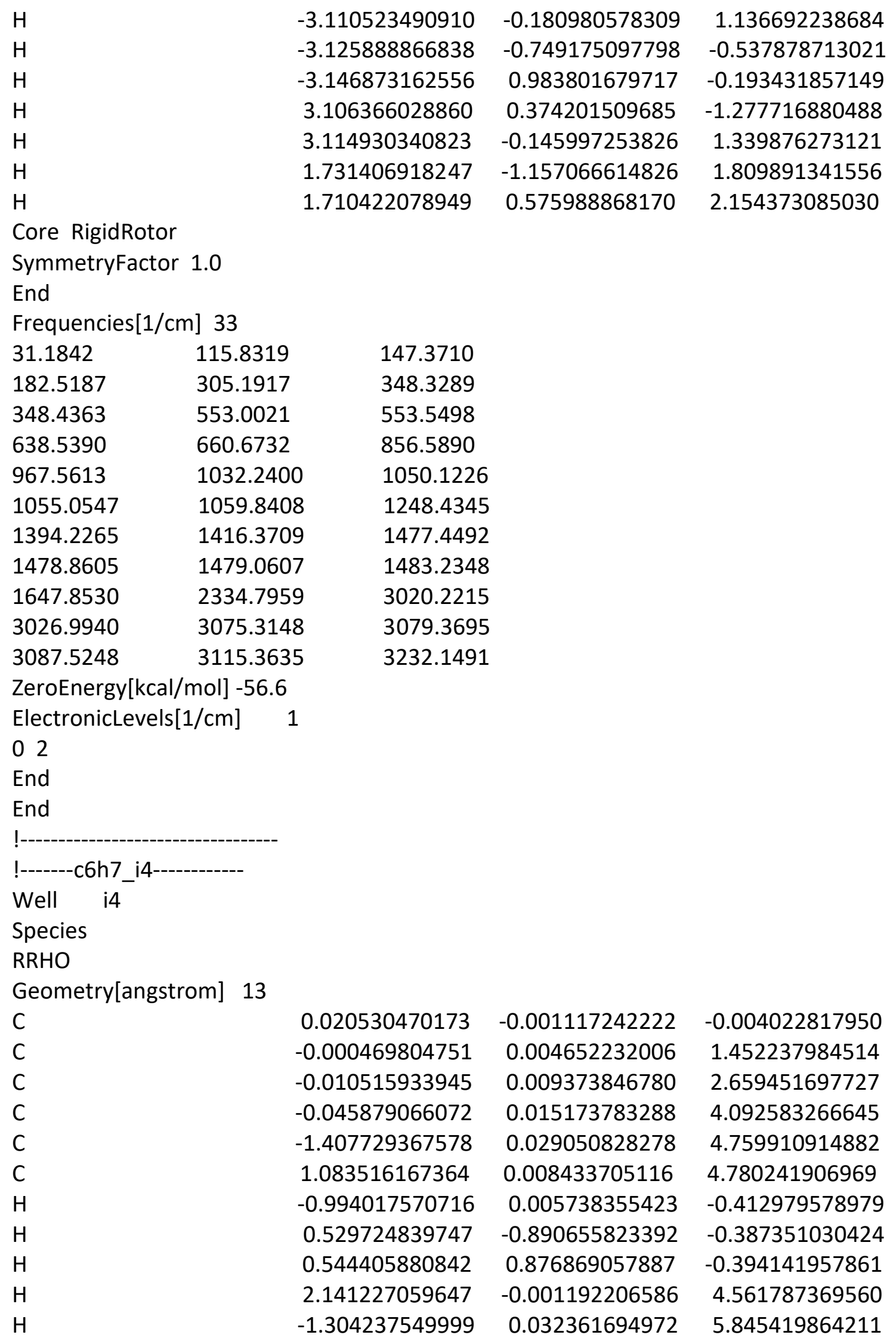




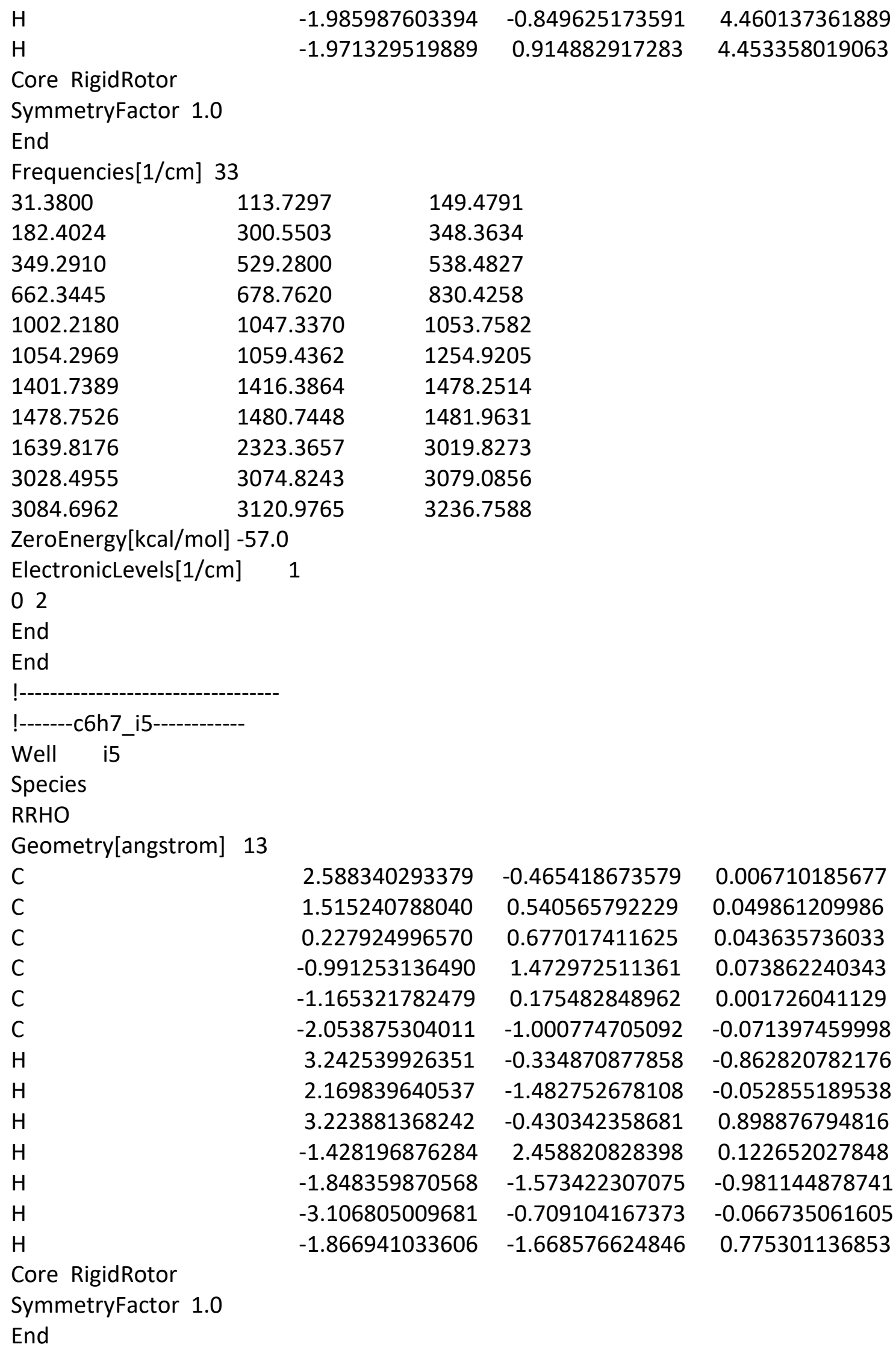


Frequencies[1/cm] 33

$\begin{array}{lll}112.0501 & 117.6767 & 135.9239 \\ 187.6721 & 262.2818 & 288.7270 \\ 461.6761 & 493.1371 & 634.6647 \\ 745.3713 & 753.8956 & 951.2658 \\ 985.3987 & 1033.1357 & 1045.0396 \\ 1051.9461 & 1110.1456 & 1174.2203 \\ 1397.9729 & 1403.9710 & 1458.5241 \\ 1473.2560 & 1478.1590 & 1486.4928 \\ 1726.3058 & 1897.0678 & 2952.7157 \\ 3021.6969 & 3023.5445 & 3042.0855 \\ 3076.4427 & 3098.5260 & 3231.1066\end{array}$

ZeroEnergy[kcal/mol] -37.4

ElectronicLevels[1/cm] 1

02

End

End

!---------------------------------

!-------c6h7_i7-------------

Well i7

Species

RRHO

Geometry[angstrom] 13

C

C

0.460232104219

0.004116193754

$-0.270558641163$

$-0.066231695487$

0.013051960986

1.081209458693

$-0.487572562989$

0.020214452796

2.240376460279

$-0.990517006123$

0.026280992600

3.460647295436

$-1.295988422818$

0.039368097803

4.734221423089

$-2.692784720848$

0.005893445520

5.305449853039

1.554660775035

$-0.063836545747$

$-0.277449781809$

0.072464851413

$-0.847036268395$

$-0.840797837100$

0.181938706838

0.917173767654

$-0.807559099195$

$-0.486029476760$

0.077698670811

5.469390880592

$-3.444959044153$

$-0.031599873376$

4.516799968484

$-2.823095866769$

$-0.867837408162$

5.953562984293

$-2.878068636096$

0.892219514087

5.922323037291

Core RigidRotor

SymmetryFactor 1.0

End

Frequencies[1/cm] 33

17.2758

140.7434

368.9529

660.0272
43.1626

171.8383

418.2412

718.6464
114.7958

304.8717

513.5602

930.9224 


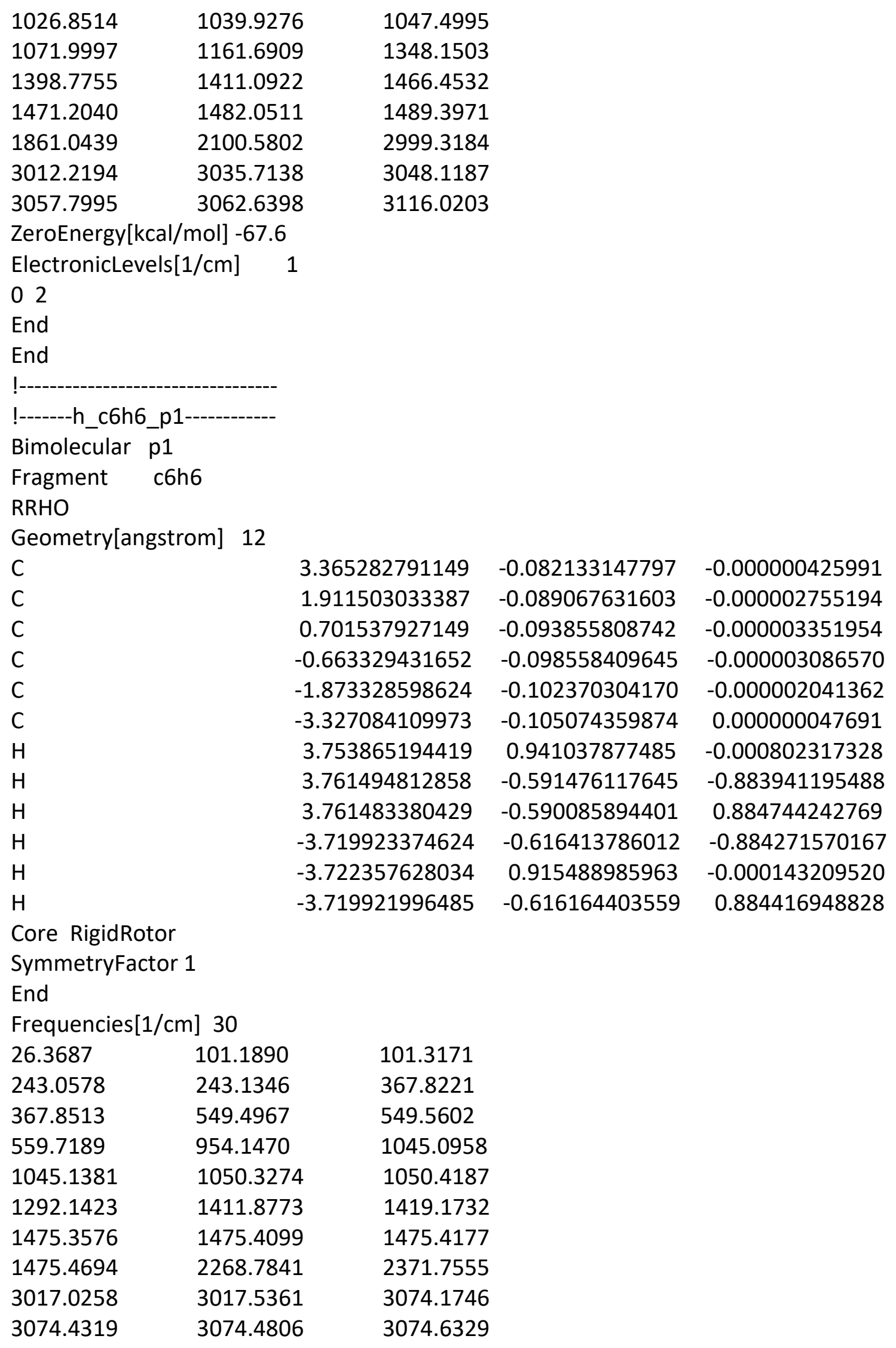




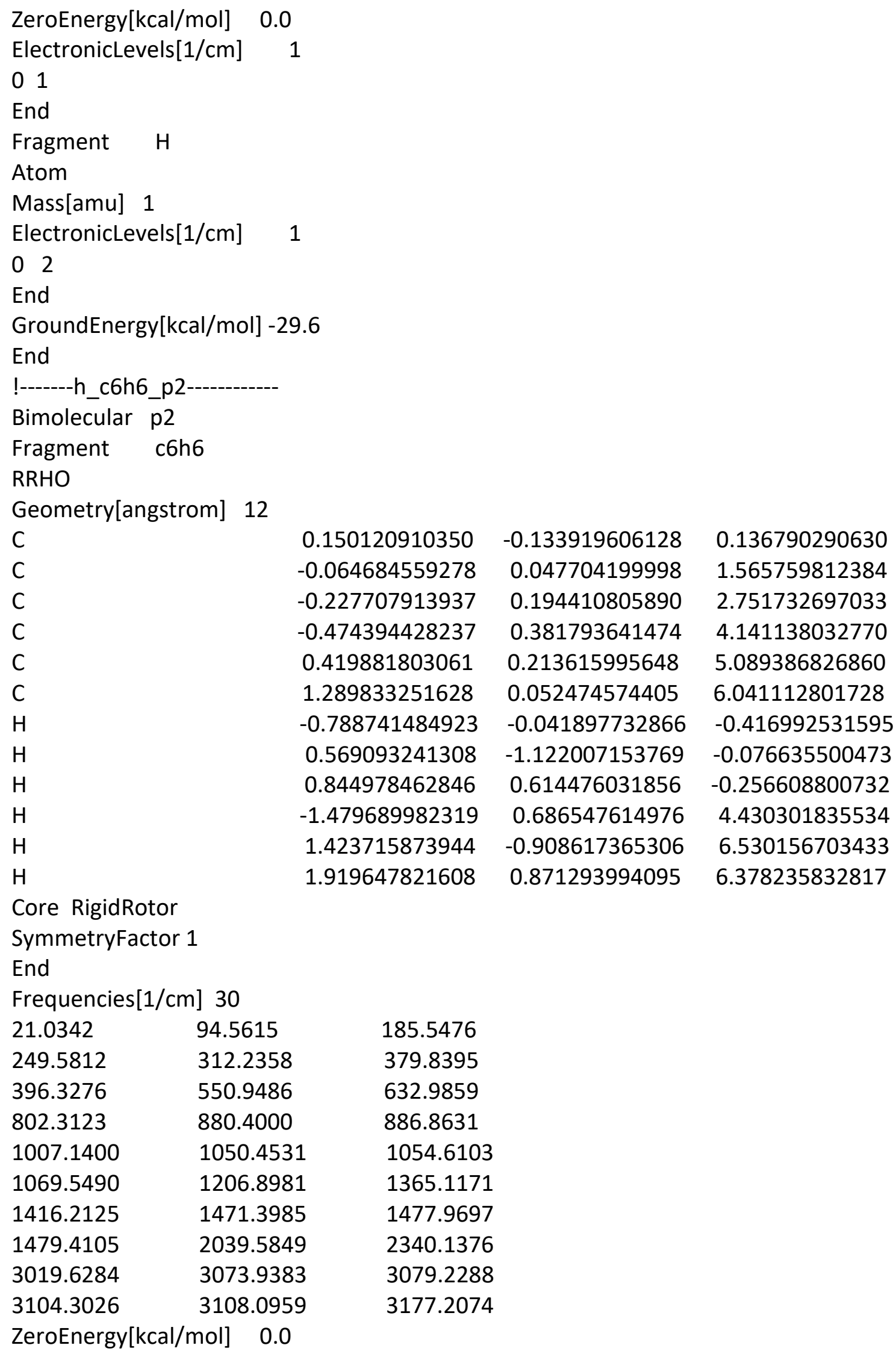

21.0342

249.5812

396.3276

802.3123

1007.1400

1069.5490

1416.2125

1479.4105

3019.6284

94.5615

312.2358

550.9486

880.4000

1050.4531

1206.8981

1471.3985

2039.5849

3073.9383

3108.0959

ZeroEnergy[kcal $/ \mathrm{mol}] \quad 0.0$

0.150120910350

$-0.064684559278$

$-0.227707913937$

$-0.474394428237$

0.419881803061

1.289833251628

$-0.788741484923$

0.569093241308

0.844978462846

$-1.479689982319$

1.423715873944

1.919647821608

$-0.133919606128$ 0.047704199998

0.194410805890

0.381793641474

0.213615995648

0.052474574405

$-0.041897732866$

$-1.122007153769$

0.614476031856

0.686547614976

$-0.908617365306$

0.871293994095

0.136790290630

1.565759812384

2.751732697033

4.141138032770

5.089386826860

6.041112801728

$-0.416992531595$

$-0.076635500473$

$-0.256608800732$

4.430301835534

6.530156703433

6.378235832817

185.5476

379.8395

632.9859

886.8631

1054.6103

1365.1171

1477.9697

2340.1376

3079.2288

3177.2074 


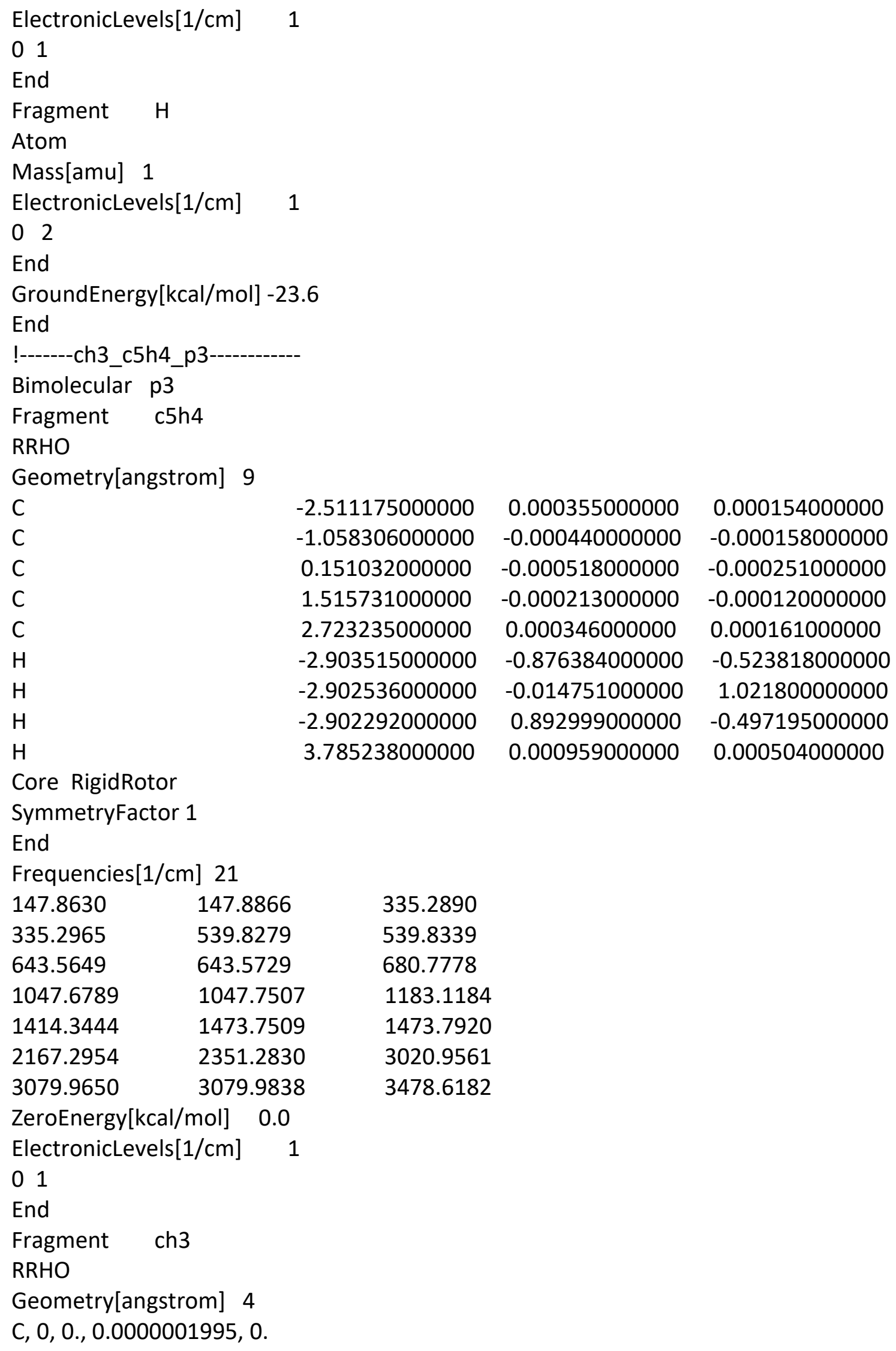

539.8339

680.7778

1183.1184

1473.7920

3020.9561

3478.6182 
$\mathrm{H}, 0,0 ., 1.0804553795,0$.

$\mathrm{H}, 0,0.9357017515,-0.5402277895,0$.

H, 0, $-0.9357017515,-0.5402277895,0$.

Core RigidRotor

SymmetryFactor 6

End

Frequencies[1/cm] 6

$\begin{array}{lll}505.1501 & 1403.0831 & 1403.0885\end{array}$

$3103.6402 \quad 3282.6573 \quad 3282.6683$

ZeroEnergy[kcal/mol] $\quad 0.0$

ElectronicLevels[1/cm] 1

02

End

GroundEnergy[kcal/mol] -36.3

End

!-------h_c6h6_p4-------------

Bimolecular p4

Fragment c6h6

RRHO

Geometry[angstrom] 12

C, 0, -0.759696, 0., 0.

C, 0, 0.12596, 1.178158, 0 .

C, $0,0.12596,-1.178158,0$.

C, $0,1.402657,0.737542,0$.

C, $0,1.402657,-0.737542,0$.

C, $0,-2.100626,0 ., 0$.

$\mathrm{H}, 0,-0.218274,2.202502,0$.

$\mathrm{H}, 0,-0.218274,-2.202502,0$.

$\mathrm{H}, 0,2.294557,1.34981,0$.

$H, 0,2.294557,-1.34981,0$.

$\mathrm{H}, 0,-2.667015,-0.924873,0$.

H, 0, -2.667015, 0.924873, 0.

Core RigidRotor

SymmetryFactor 2

End

Frequencies[1/cm] 30

208.9659

636.8016

779.2379

909.0420

962.0616

1102.3399

1334.2716

1531.9813
$344.4463 \quad 492.6127$

$679.7937 \quad 691.3194$

$790.9522 \quad 807.8704$

$929.8510 \quad 943.5150$

$962.1712 \quad 999.7109$

$1104.8600 \quad 1252.7553$

$1368.3560 \quad 1452.5919$

$1616.7669 \quad 1699.6352$ 


\begin{tabular}{|c|c|c|c|}
\hline 3135.3788 & \multicolumn{2}{|c|}{3196.5225} & 3206.3028 \\
\hline 3220.5810 & \multicolumn{2}{|c|}{3223.6554} & 3229.6272 \\
\hline \multicolumn{2}{|c|}{ ZeroEnergy[kcal/mol] } & 0.0 & \\
\hline \multicolumn{2}{|c|}{ ElectronicLevels[1/cm] } & 1 & \\
\hline \multicolumn{4}{|c|}{01} \\
\hline \multicolumn{4}{|l|}{ End } \\
\hline Fragment & \multicolumn{3}{|l|}{$\mathrm{H}$} \\
\hline \multicolumn{4}{|l|}{ Atom } \\
\hline \multicolumn{4}{|c|}{ Mass[amu] 1} \\
\hline \multicolumn{4}{|c|}{ ElectronicLevels[1/cm] } \\
\hline \multicolumn{4}{|c|}{02} \\
\hline \multicolumn{4}{|c|}{ End } \\
\hline \multicolumn{4}{|c|}{ GroundEnergy $[\mathrm{kcal} / \mathrm{mol}]-66.0$} \\
\hline \multicolumn{4}{|c|}{ End } \\
\hline \multicolumn{4}{|c|}{ !-------h_c6h6_p5------------ } \\
\hline \multicolumn{4}{|c|}{ Bimolecular p5 } \\
\hline \multicolumn{4}{|c|}{ Fragment c6h6 } \\
\hline \multicolumn{4}{|c|}{$\mathrm{RRHO}$} \\
\hline \multicolumn{4}{|c|}{ Geometry[angstrom] 12} \\
\hline $\mathrm{C}$ & 0. & 0. & 0. \\
\hline C & 0. & 0. & 1.47153 \\
\hline C & 0.78173 & 0. & 2.5034 \\
\hline C & 2.10193 & 0. & 3.17532 \\
\hline C & 3.56938 & 0. & 3.01702 \\
\hline C & 1.03514 & 0. & 3.9375 \\
\hline $\mathrm{H}$ & -0.50203 & -0.88218 & -0.41332 \\
\hline $\mathrm{H}$ & -0.502 & 0.8822 & -0.41332 \\
\hline $\mathrm{H}$ & 0.61428 & 0. & 4.93152 \\
\hline $\mathrm{H}$ & 3.88801 & -0.87956 & 2.44899 \\
\hline $\mathrm{H}$ & 4.07726 & 0. & 3.98439 \\
\hline $\mathrm{H}$ & 3.88801 & 0.87957 & 2.449 \\
\hline \multicolumn{4}{|c|}{ Core RigidRotor } \\
\hline \multicolumn{4}{|c|}{ SymmetryFactor 1} \\
\hline \multicolumn{4}{|c|}{ End } \\
\hline \multicolumn{4}{|c|}{ Frequencies[1/cm] 30} \\
\hline 130.0531 & 135 & 3324 & 190.8871 \\
\hline 263.0030 & 319. & 8387 & 437.2043 \\
\hline 556.4633 & 633. & 7750 & 686.1637 \\
\hline 751.9313 & 768. & 3806 & 884.7891 \\
\hline 982.5922 & 100 & .6810 & 1041.1961 \\
\hline 1047.1792 & 113 & .4582 & 1358.9142 \\
\hline 1403.2113 & 147 & .2691 & 1477.7809 \\
\hline 1521.1988 & 179 & .8244 & 2102.2295 \\
\hline 3026.7295 & 307 & .1934 & 3080.1691 \\
\hline
\end{tabular}




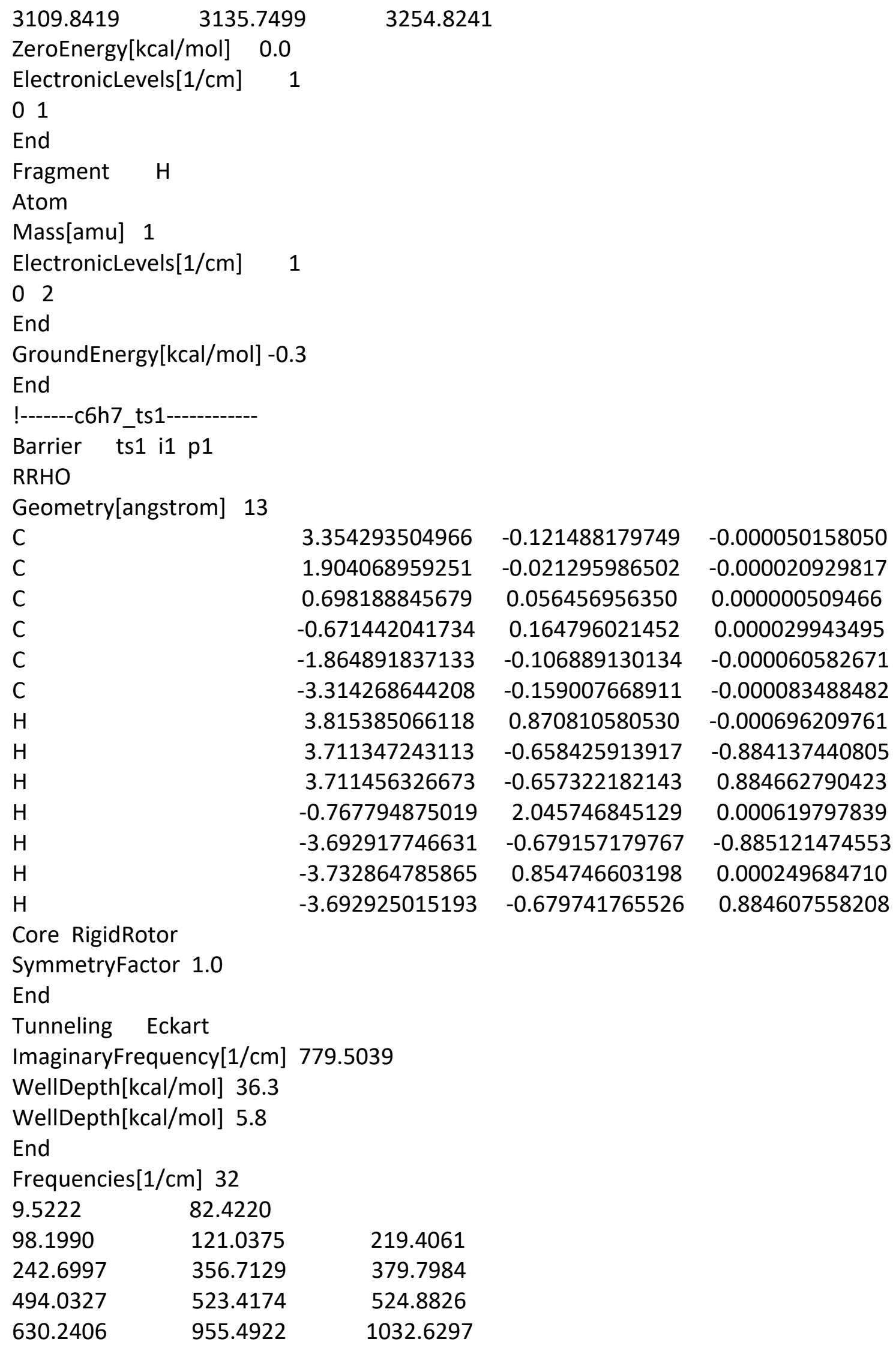

$-0.000050158050$

$-0.000020929817$

0.000000509466

0.000029943495

$-0.000060582671$

$-0.000083488482$

$-0.000696209761$

$-0.884137440805$

0.884662790423

0.000619797839

$-0.885121474553$

0.000249684710

0.884607558208 


\begin{tabular}{|c|c|c|c|c|}
\hline 1048.1794 & 1049.6430 & 1051.3682 & & \\
\hline 1281.8939 & 1408.3274 & 1416.3536 & & \\
\hline 1466.6094 & 1471.9500 & 1474.9213 & & \\
\hline 1475.0661 & 2190.7028 & 2347.3999 & & \\
\hline 3006.6320 & 3019.4471 & 3062.4890 & & \\
\hline 3076.9987 & 3077.9574 & \multirow[t]{2}{*}{3077.9833} & & \\
\hline \multicolumn{4}{|c|}{ ZeroEnergy[kcal/mol] -23.8} & \\
\hline \multicolumn{5}{|c|}{ ElectronicLevels[1/cm] 1} \\
\hline \multicolumn{5}{|c|}{02} \\
\hline \multicolumn{5}{|l|}{ End } \\
\hline \multicolumn{5}{|c|}{ 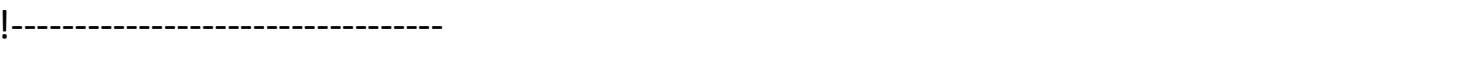 } \\
\hline \multicolumn{5}{|c|}{ !-------c6h7_ts2----------- } \\
\hline \multicolumn{5}{|c|}{ Barrier ts2 i1 i5 } \\
\hline \multicolumn{5}{|c|}{ RRHO } \\
\hline \multicolumn{5}{|c|}{ Geometry[angstrom] 13} \\
\hline \multicolumn{2}{|c|}{ C } & 0.005362241942 & 0.093981437319 & 0.157797125675 \\
\hline \multicolumn{2}{|l|}{ c } & -0.027733322788 & -0.363469594707 & 1.543697802639 \\
\hline C & & -0.002314163632 & -0.282467053334 & 2.793355420465 \\
\hline C & & 0.133900719161 & 0.772145908671 & 4.186936531538 \\
\hline C & & 0.285851341276 & 2.046366825613 & 4.905950951979 \\
\hline C & & -0.011175272132 & -0.525535481482 & 4.196225135863 \\
\hline H & & 0.835811749107 & -0.353341715303 & -0.399077066214 \\
\hline $\mathrm{H}$ & & 0.127901702048 & 1.185578416392 & 0.105972367720 \\
\hline $\mathrm{H}$ & & -0.918295931954 & -0.155353059835 & -0.375625050389 \\
\hline $\mathrm{H}$ & & -0.098432365694 & -1.382615899792 & 4.857844397956 \\
\hline $\mathrm{H}$ & & 1.222551389555 & 2.538307227077 & 4.625518423981 \\
\hline $\mathrm{H}$ & & 0.283529003677 & 1.899084408805 & 5.994087488110 \\
\hline $\mathrm{H}$ & & -0.525852091985 & 2.733966580100 & 4.648270470834 \\
\hline Core Rigid & & & & \\
\hline SymmetryF & 1.0 & & & \\
\hline End & & & & \\
\hline Tunneling & & & & \\
\hline ImaginaryF & $\mathrm{ncy}[1 / \mathrm{cm}] \epsilon$ & 630.2406 & & \\
\hline WellDepth & nol] 28.9 & & & \\
\hline WellDepth & nol] 6.2 & & & \\
\hline End & & & & \\
\hline Frequencie & n] 32 & & & \\
\hline 82.3594 & 105.5250 & & & \\
\hline 122.1335 & 198.4387 & 224.3082 & & \\
\hline 240.1000 & 418.0151 & 423.7397 & & \\
\hline 711.5693 & 778.0967 & 870.9375 & & \\
\hline 1013.0451 & 1037.2757 & 1041.1812 & & \\
\hline 1055.3997 & 1091.7651 & 1250.0055 & & \\
\hline 1394.2660 & 1408.9592 & 1463.4319 & & \\
\hline
\end{tabular}




\begin{tabular}{|c|c|c|c|c|c|}
\hline 1467.1878 & 147 & .4554 & 1480.3277 & & \\
\hline 1792.2566 & 198 & 3.8616 & 2972.9807 & & \\
\hline 2987.6951 & 303 & 2.6021 & 3053.0536 & & \\
\hline 3057.5721 & 307 & 2.1124 & 3134.1232 & & \\
\hline ZeroEnergy & $/ \mathrm{mol}]$ & -31.2 & & & \\
\hline ElectronicL & $1 / \mathrm{cm}$ & & & & \\
\hline 02 & & & & & \\
\hline End & & & & & \\
\hline !-------------- & & & & & \\
\hline !-------c6h7 & --.-. & & & & \\
\hline Barrier $t$ & & & & & \\
\hline RRHO & & & & & \\
\hline Geometry[ & rom] & 13 & & & \\
\hline C & & & 0.242224968454 & -0.240058858005 & 0.269187706748 \\
\hline C & & & -0.017080324716 & 0.042236198094 & 1.674008521886 \\
\hline c & & & -0.217281895777 & 0.268840451313 & 2.841422879656 \\
\hline c & & & -0.499095074357 & 0.559921871799 & 4.206458748191 \\
\hline C & & & 0.277342294480 & 0.236769076892 & 5.214223015574 \\
\hline C & & & 1.074306468056 & -0.100233302853 & 6.196898414738 \\
\hline $\mathrm{H}$ & & & -0.688673362579 & -0.272863796823 & -0.304432336759 \\
\hline $\mathrm{H}$ & & & 0.744965172086 & -1.204216773736 & 0.149133211792 \\
\hline $\mathrm{H}$ & & & 0.882386313170 & 0.527804053837 & -0.176153653794 \\
\hline $\mathrm{H}$ & & & -1.428092312051 & 1.088978912196 & 4.419814715182 \\
\hline $\mathrm{H}$ & & & 2.716138676854 & -1.088032461528 & 5.302634857032 \\
\hline$H$ & & & 0.929095041533 & -1.025248942789 & 6.747059981838 \\
\hline $\mathrm{H}$ & & & 1.814117018220 & 0.593853574102 & 6.584633940206 \\
\hline
\end{tabular}

Core RigidRotor

SymmetryFactor 1.0

End

Tunneling Eckart

ImaginaryFrequency[1/cm] 428.063

WellDepth $[\mathrm{kcal} / \mathrm{mol}] 39.3$

WellDepth[kcal/mol] 2.8

End

Frequencies[1/cm] 32

18.0043

85.9595

154.1788

210.2840

252.2244

303.2514

323.9833

400.6462

408.0093

550.9847

676.1447

796.9069

887.0370

900.0315

1013.3272

1050.4432

1053.8759

1059.2785

1204.3406

1356.5699

1416.1685

1465.4872

1477.5033

1479.0971

2000.9822

2340.3094 


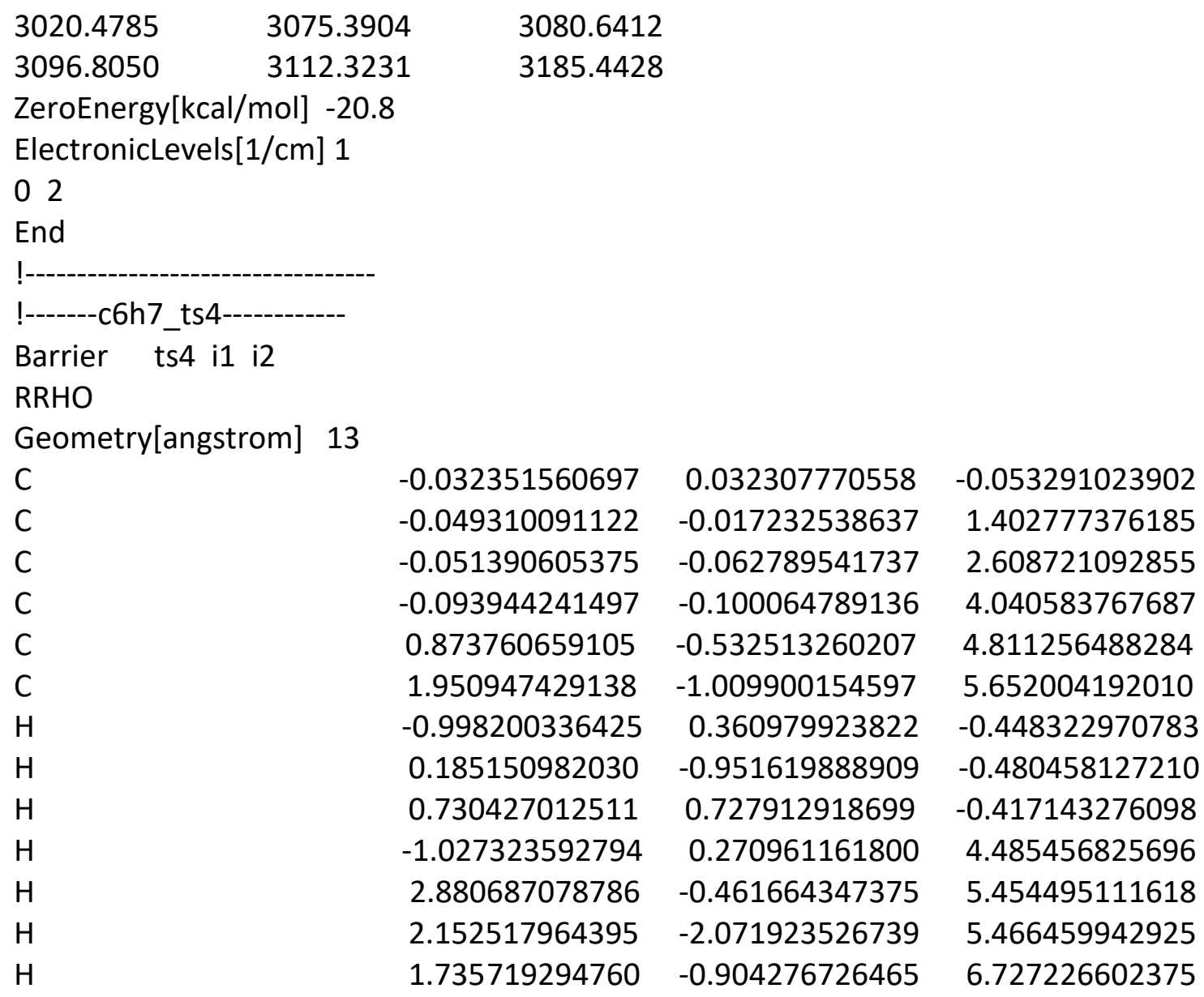

Core RigidRotor

SymmetryFactor 1.0

End

Tunneling Eckart

ImaginaryFrequency[1/cm] 274.4071

WellDepth[kcal/mol] 6.0

WellDepth[kcal/mol] 6.3

End

Frequencies[1/cm] 32

12.7785

104.9059

302.9418

734.4959

992.0213

1054.6605

1404.2166

1467.1882

1805.2506

2973.2913

3042.8643
20.4842

$194.1049 \quad 288.0033$

$388.8273 \quad 493.8290$

$788.0058 \quad 898.2066$

$1026.0294 \quad 1050.5272$

$1183.1481 \quad 1307.5790$

$1416.6554 \quad 1449.0422$

$1479.0772 \quad 1479.8759$

$2329.2743 \quad 2942.5693$

$3007.6455 \quad 3017.2529$

$3070.2944 \quad 3075.8048$ 
ZeroEnergy[kcal $/ \mathrm{mol}]-54.1$

ElectronicLevels[1/cm] 1

02

End

!-

Barrier ts5 i2 p1

RRHO

Geometry[angstrom] 13

C

C

0.937899811208

$-0.069673071546$

$-0.547251029879$

0.240324905137

$-0.034116117508$

0.727627255079

C

$-0.344172889993$

$-0.006298639147$

1.784878853286

$-0.991989001131$

0.031602352964

2.995880069819

$-1.849905242966$

$-0.050612096206$

3.864992101927

$-2.669107988353$

$-0.062998994271$

5.061722196449

1.980528917636

0.241612558812

$-0.431963365151$

0.933410660904

$-1.078989810543$

$-0.969935787918$

0.465678825949

0.599769428953

$-1.272686669059$

0.463969279749

0.623980299917

4.033442661336

$-3.523196124193$

0.614102502408

4.965335371672

$-3.055532901166$

$-1.065653214888$

5.268080436243

$-2.081079254488$

0.257274804387

5.930380905674

Core RigidRotor

SymmetryFactor 1.0

End

Tunneling Eckart ImaginaryFrequency[1/cm] 779.5028

WellDepth $[\mathrm{kcal} / \mathrm{mol}] 36.6$

WellDepth[kcal/mol] 5.8

End

Frequencies[1/cm] 32

9.9344

98.2007

242.7016

494.0324

630.2405

1048.1821

1281.8939

1466.6097

1475.0669

3006.6300

3076.9875

ZeroEnergy[kcal/mol] -23.8

ElectronicLevels[1/cm] 1

3077.9461

\subsection{7}

121.0391

356.7140

523.4174

955.4922

1049.6461

1408.3281

1471.9503

2190.7029

3019.4364

219.4076

379.7997

524.8821

1032.6304

1051.3693

1416.3552

1474.9221

2347.3998

3062.4870

3077.9812 


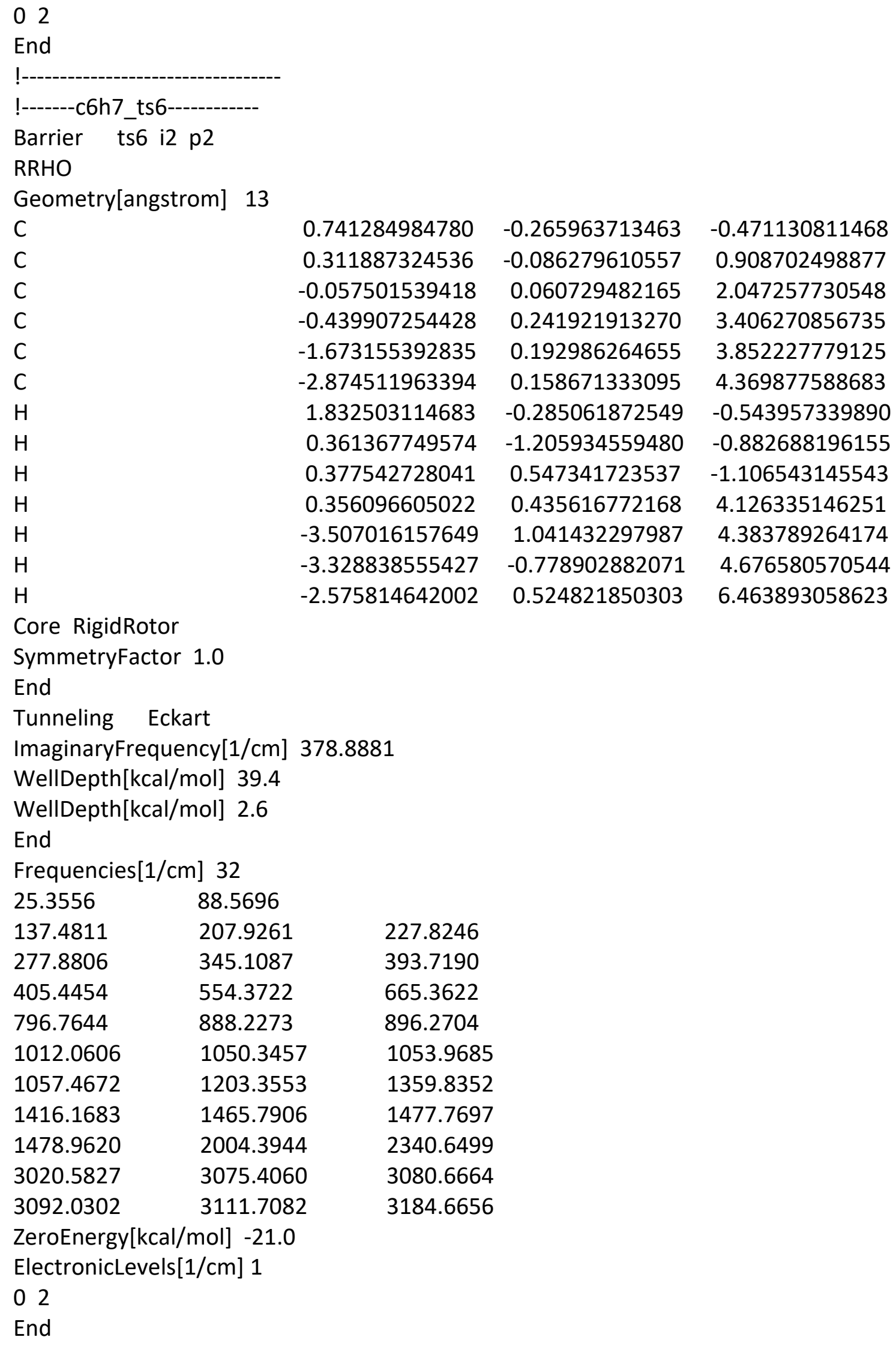

227.8246

393.7190

665.3622

896.2704

1053.9685

1359.8352

1477.7697

2340.6499

3080.6664

3184.6656 


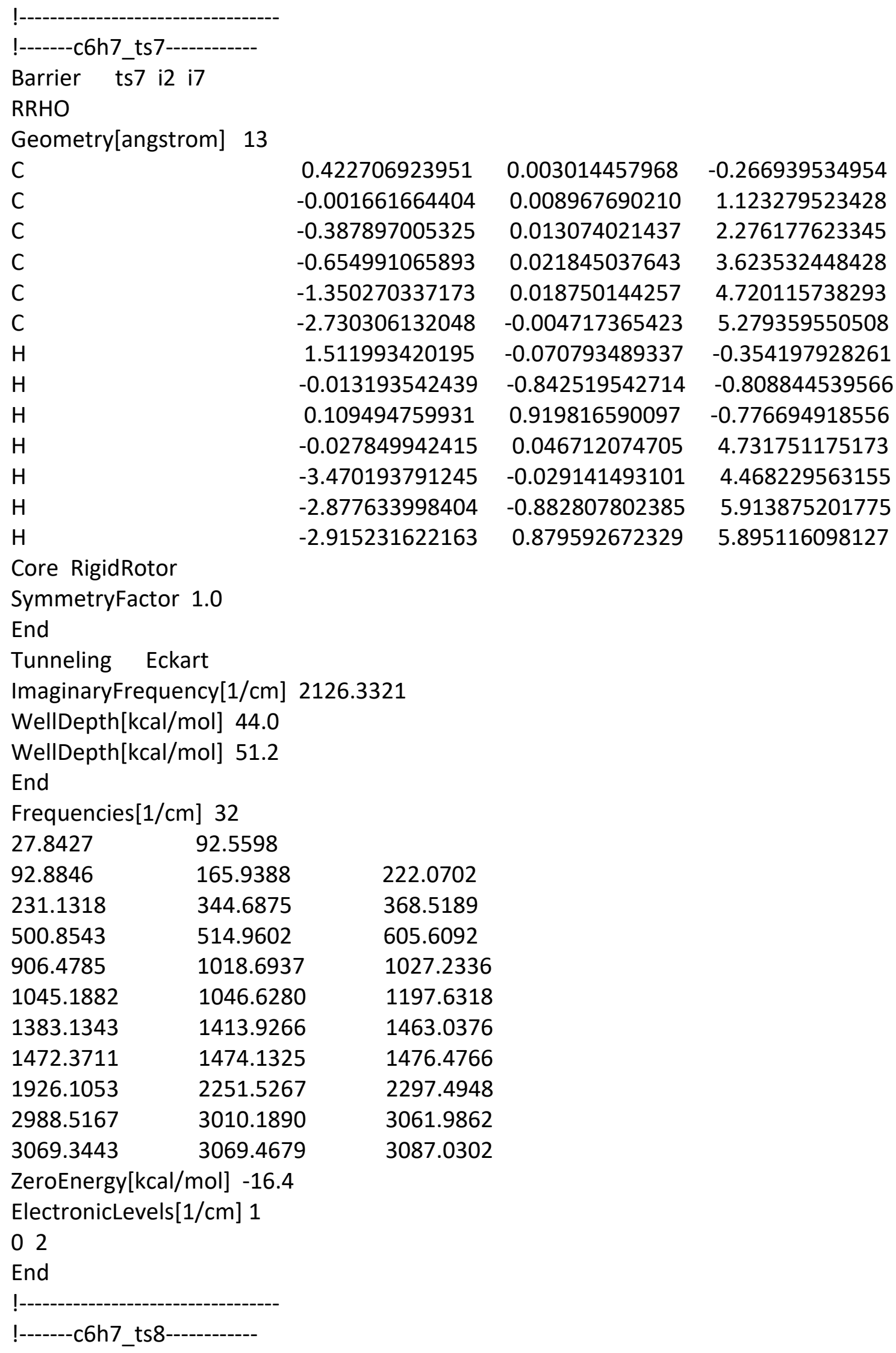




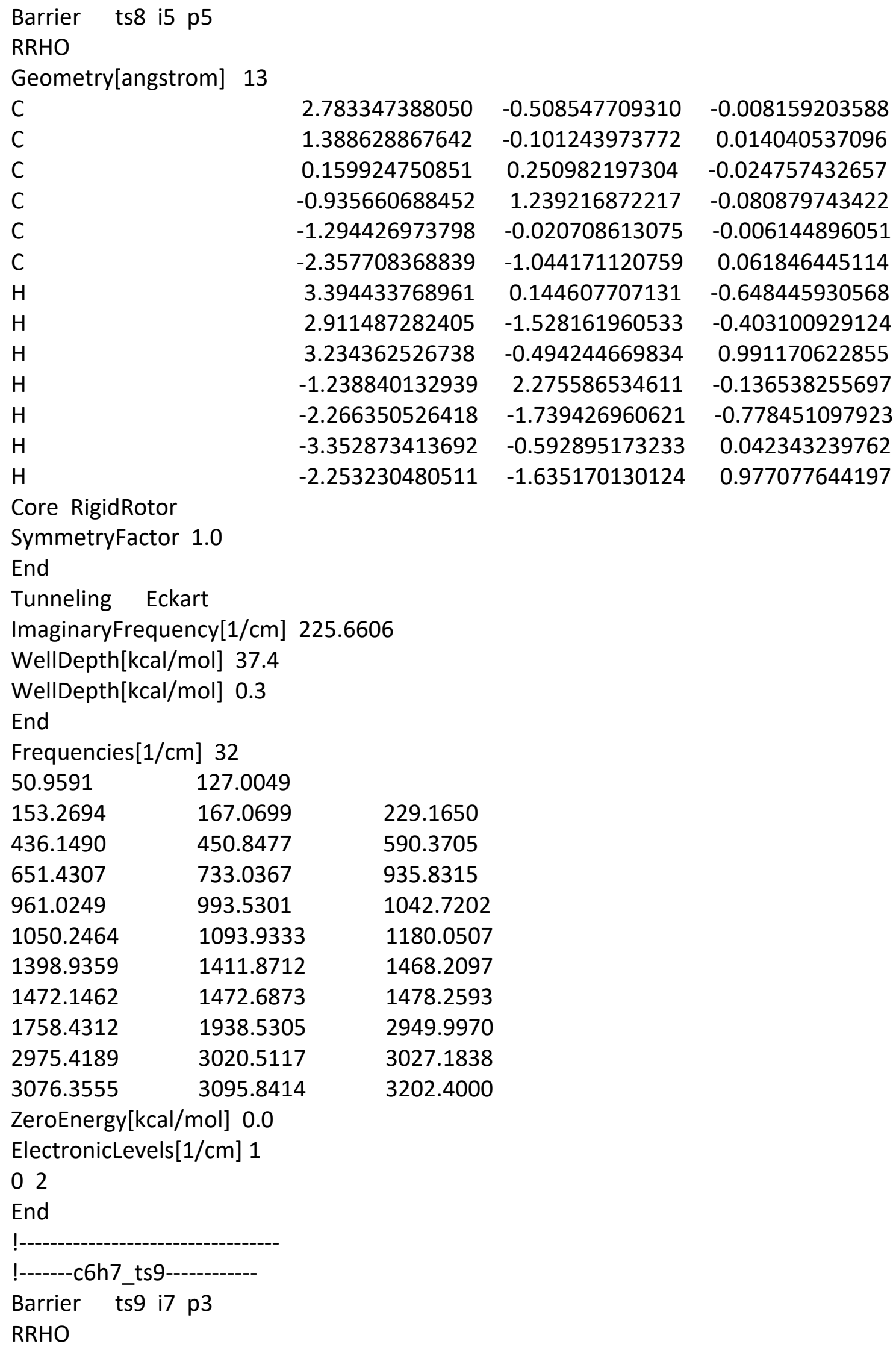




\begin{tabular}{llll}
\multicolumn{4}{l}{ Geometry[angstrom] 13} \\
C & 0. & 0. & 0. \\
C & 0. & 0. & 1.4507 \\
C & 0.02807 & 0. & 2.68377 \\
C & 0.0023 & -0.00246 & 4.00338 \\
C & 0.17976 & 0.00537 & 5.30104 \\
C & -1.34157 & -0.07356 & 6.74649 \\
H & 1.0182 & -0.04978 & -0.40401 \\
H & -0.55513 & -0.85739 & -0.39587 \\
H & -0.46826 & 0.9084 & -0.39376 \\
H & 1.20063 & 0.05702 & 5.69237 \\
H & -2.32803 & -0.12341 & 6.28436 \\
H & -1.21427 & -0.94943 & 7.39231 \\
H & -1.30396 & 0.80948 & 7.39399
\end{tabular}

Core RigidRotor

SymmetryFactor 1.0

End

Tunneling Eckart

ImaginaryFrequency[1/cm] 383.54

WellDepth $[\mathrm{kcal} / \mathrm{mol}] 38.1$

WellDepth[kcal/mol] 6.8

End

Frequencies[1/cm] 32

20.2998 30.7871

68.4182 149.0064

335.1022 337.9133

179.3176

434.2015

497.1515

405.1173

625.0096

661.1257

539.1824

766.3374

1044.5088

684.0507

1183.3311

1408.8048

1046.5764

1416.6533

1472.4251

1413.5738

2063.3872

2294.2366

1473.6290

3073.1543

3076.3751

3016.8723

3259.2311

3270.9656

ZeroEnergy $[\mathrm{kcal} / \mathrm{mol}]-29.5$

ElectronicLevels[1/cm] 1

02

End

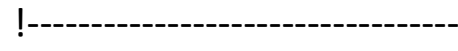

!-------c6h7_ts10-------------

Barrier ts10 i7 p1

RRHO

Geometry[angstrom] 13

C

$\begin{array}{lll}0.161854183164 & 0.010854868521 & -0.070225675459\end{array}$ 


$\begin{array}{lrrr}\text { C } & -0.029656463391 & 0.000441775549 & 1.369890177347 \\ \mathrm{C} & -0.186018752608 & -0.008168358136 & 2.570943994538 \\ \mathrm{C} & -0.368569340289 & -0.017986838469 & 3.918523383880 \\ \mathrm{C} & -0.457264368073 & -0.025152324500 & 5.132943098105 \\ \mathrm{C} & -0.857302999737 & -0.040035506296 & 6.535924004793 \\ \mathrm{H} & 1.226388600968 & 0.035506334737 & -0.323106542877 \\ \mathrm{H} & -0.271677291897 & -0.881870753813 & -0.531334293916 \\ \mathrm{H} & -0.310847775945 & 0.887438120583 & -0.523750040282 \\ \mathrm{H} & 1.603854843639 & 0.018820973972 & 5.547810479220 \\ \mathrm{H} & -1.948199975140 & -0.064602449889 & 6.617604677483 \\ \mathrm{H} & -0.454485627334 & -0.916160018613 & 7.051142086479 \\ \mathrm{H} & -0.493664037850 & 0.848663182766 & 7.058692650467\end{array}$

Core RigidRotor

SymmetryFactor 1.0

End

Tunneling Eckart

ImaginaryFrequency[1/cm] 470.8111

WellDepth $[\mathrm{kcal} / \mathrm{mol}] 41.0$

WellDepth $[\mathrm{kcal} / \mathrm{mol}] 3.0$

End

Frequencies[1/cm] 32

$\begin{array}{lll}18.1137 & 98.9648 & \\ 99.9033 & 139.1534 & 252.3376 \\ 256.0663 & 373.9052 & 378.7743 \\ 404.4285 & 529.5211 & 532.4778 \\ 570.2354 & 950.2169 & 1038.9576 \\ 1047.1016 & 1047.8548 & 1055.5166 \\ 1291.5473 & 1410.4846 & 1417.0898 \\ 1472.1484 & 1473.6190 & 1473.8209 \\ 1477.1841 & 2230.5383 & 2332.4403 \\ 3018.2160 & 3024.1028 & 3075.7615 \\ 3076.7164 & 3084.9788 & 3087.2106\end{array}$

ZeroEnergy[kcal/mol] -26.6

ElectronicLevels[1/cm] 1

02

End

!---------------------------

!------c6h7_ts11----------

Barrier ts11 i3 p3

RRHO

Geometry[angstrom] 13

C

C

$-0.204918284838 \quad-0.001072726293$

0.146950063174

$-0.047432105591 \quad-0.002258449178$

1.592780264945

C

$0.104396331829 \quad-0.003236173027$

2.791467362597 


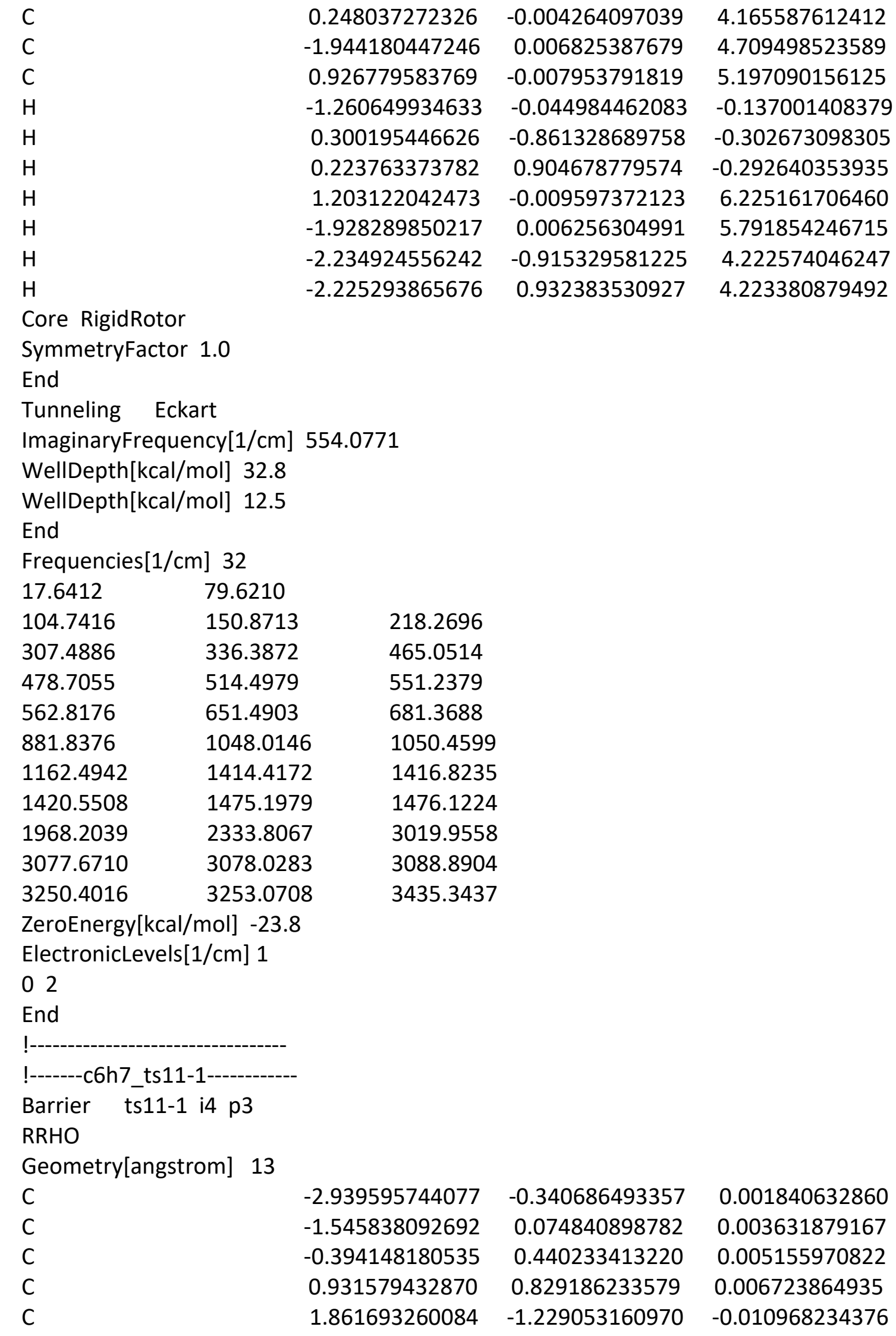




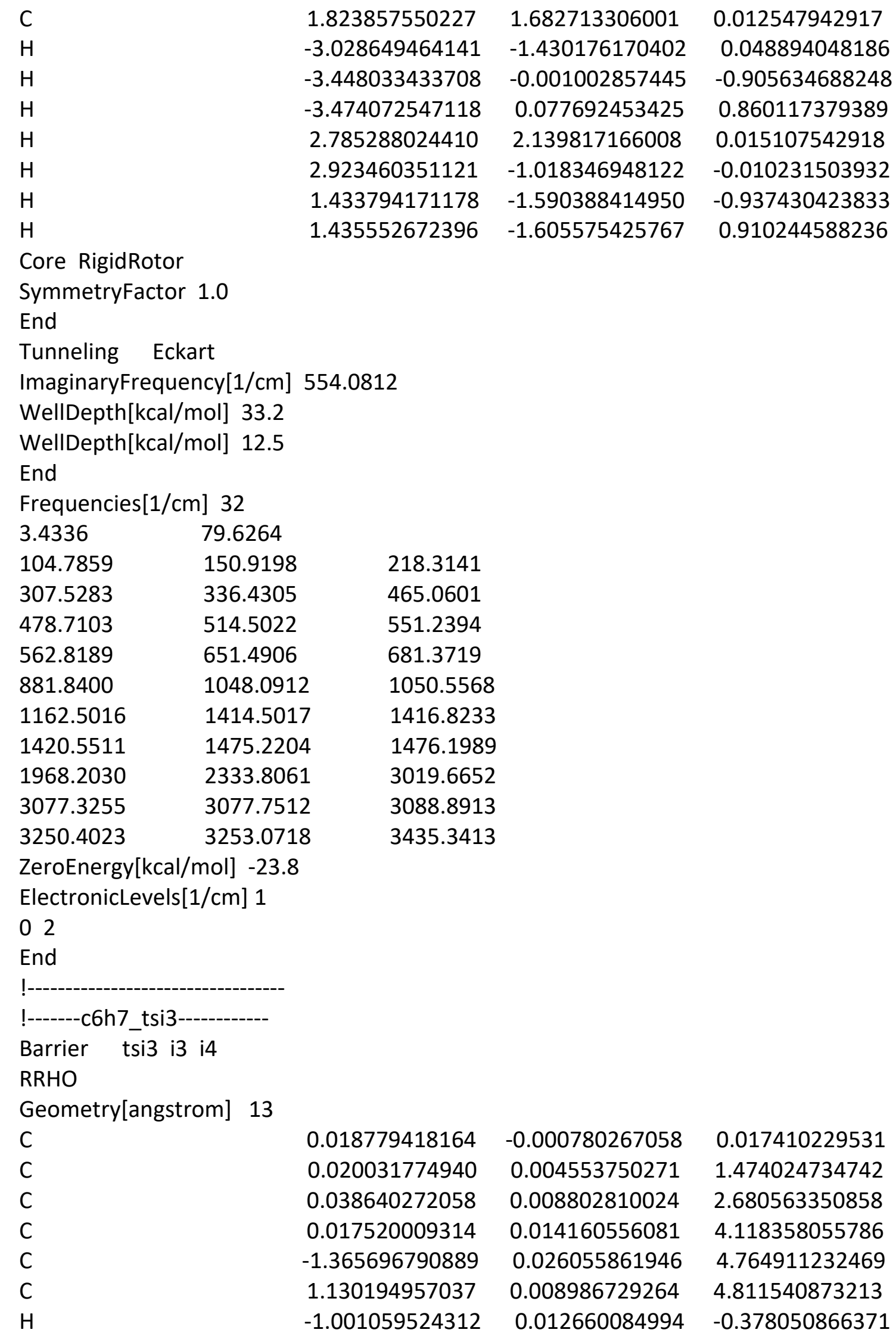




$\begin{array}{lrrr}\mathrm{H} & 0.517536665461 & -0.893101864053 & -0.373217776920 \\ \mathrm{H} & 0.543398473970 & 0.873654607701 & -0.379716740741 \\ \mathrm{H} & 2.017345565395 & 0.004988602424 & 5.399757749742 \\ \mathrm{H} & -1.286019419805 & 0.029442553667 & 5.852299718830 \\ \mathrm{H} & -1.933949362180 & -0.854218109259 & 4.450590253526 \\ \mathrm{H} & -1.921752037107 & 0.911796685572 & 4.444198185508\end{array}$

Core RigidRotor

SymmetryFactor 1.0

End

Tunneling Eckart

ImaginaryFrequency[1/cm] 722.2746

WellDepth[kcal $/ \mathrm{mol}] 4.4$

WellDepth[kcal $/ \mathrm{mol}] 4.8$

End

Frequencies[1/cm] 32

$\begin{array}{lll}31.4430 & 114.0529 & \\ 144.9136 & 172.0926 & 302.6243 \\ 348.3774 & 365.4688 & 538.9187 \\ 556.4359 & 595.5996 & 658.8908 \\ 948.3156 & 1032.4502 & 1050.2187 \\ 1050.6063 & 1055.7449 & 1216.3478 \\ 1390.7300 & 1416.2429 & 1478.2955 \\ 1478.9833 & 1479.3696 & 1480.2774 \\ 1645.2332 & 2327.7524 & 3019.4369 \\ 3025.7126 & 3074.2315 & 3078.2315 \\ 3084.8459 & 3121.3269 & 3435.8852\end{array}$

ZeroEnergy $[\mathrm{kcal} / \mathrm{mol}]-52.2$

ElectronicLevels[1/cm] 1

02

End

!--------------------------

!-----c6h7_ts23--------

Barrier ts23 i3 i5

RRHO

Geometry[angstrom] 13

C

C

$\begin{array}{ll}2.821517516816 & -0.487841744900\end{array}$

0.047174428763

1.364161026313

$-0.544615985066$

0.052372390260

C

0.176510110074

$-0.162409884931$

$-0.023155240944$

C

$-0.900016083151$

1.203303676738

$-0.281941822817$

$-1.245623772585$

$-0.035193702301$

$-0.051792319768$

C

$-2.405906439357$

$-0.954672025922$

0.115800956424

3.172030936587

0.536803102374

$-0.143593931562$

$\mathrm{H}$

3.250867159621

$-1.130087853127$

$-0.729742115962$

$\mathrm{H}$

3.244319090900

$-0.804387235381$

1.007098085029 


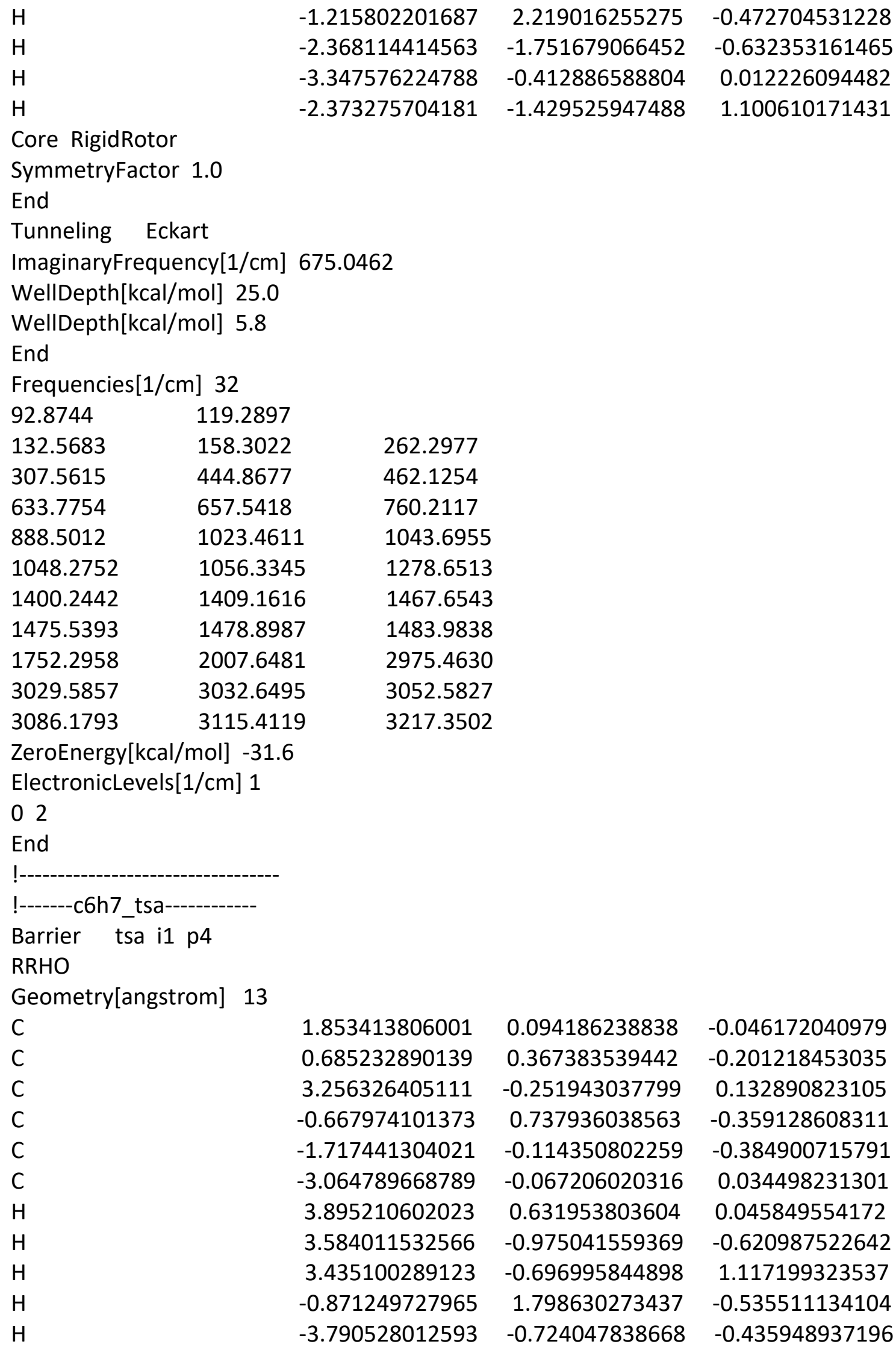




\begin{tabular}{|c|c|c|c|c|}
\hline $\mathrm{H}$ & & -3.456377848448 & \multirow{2}{*}{$\begin{array}{r}0.716582534192 \\
-0.972834324779\end{array}$} & \multirow{2}{*}{$\begin{array}{l}0.688503438531 \\
0.468273040730\end{array}$} \\
\hline $\mathrm{H}$ & & -2.192802861763 & & \\
\hline \multicolumn{5}{|c|}{ Core RigidRotor } \\
\hline \multicolumn{5}{|c|}{ SymmetryFactor 1.0} \\
\hline \multicolumn{5}{|c|}{ End } \\
\hline \multicolumn{5}{|c|}{ Tunneling Eckart } \\
\hline \multicolumn{5}{|c|}{ ImaginaryFrequency[1/cm] 1827.4491} \\
\hline \multicolumn{5}{|c|}{ WellDepth[kcal/mol] 42.6} \\
\hline \multicolumn{5}{|c|}{ WellDepth[kcal/mol] 48.5} \\
\hline \multicolumn{5}{|c|}{ End } \\
\hline \multicolumn{5}{|c|}{ Frequencies[1/cm] 32} \\
\hline 28.7984 & 100.8061 & & & \\
\hline 142.2805 & 228.9166 & 265.1482 & & \\
\hline 342.8613 & 429.1444 & 506.8345 & & \\
\hline 551.8081 & 794.1817 & 803.0838 & & \\
\hline 895.5138 & 1016.4239 & 1046.8289 & & \\
\hline 1053.8547 & 1084.4848 & 1145.0093 & & \\
\hline 1209.9182 & 1316.8338 & 1415.3530 & & \\
\hline 1465.7125 & 1477.1273 & 1479.1986 & & \\
\hline 1588.8359 & 2165.2962 & 2304.5384 & & \\
\hline 3014.0879 & 3031.4938 & 3043.3246 & & \\
\hline 3064.8769 & 3074.5144 & 3168.7704 & & \\
\hline \multicolumn{5}{|c|}{ ZeroEnergy[kcal/mol] - -17.5} \\
\hline \multicolumn{5}{|c|}{ ElectronicLevels[1/cm] 1} \\
\hline \multicolumn{5}{|c|}{02} \\
\hline \multicolumn{5}{|l|}{ End } \\
\hline \\
\hline & \multicolumn{4}{|c|}{ !-----c6h7_tsa1-------- } \\
\hline Barrier ts & $2 \mathrm{p} 4$ & & & \\
\hline \multicolumn{5}{|c|}{$\mathrm{RRHO}$} \\
\hline \multicolumn{5}{|c|}{ Geometry[angstrom] 13} \\
\hline C & & 1.853413806001 & 0.094186238838 & -0.046172040979 \\
\hline C & & 0.685232890139 & 0.367383539442 & -0.201218453035 \\
\hline c & & 3.256326405111 & -0.251943037799 & 0.132890823105 \\
\hline C & & -0.667974101373 & 0.737936038563 & -0.359128608311 \\
\hline C & & -1.717441304021 & -0.114350802259 & -0.384900715791 \\
\hline C & & -3.064789668789 & -0.067206020316 & 0.034498231301 \\
\hline $\mathrm{H}$ & & 3.895210602023 & 0.631953803604 & 0.045849554172 \\
\hline $\mathrm{H}$ & & 3.584011532566 & -0.975041559369 & -0.620987522642 \\
\hline $\mathrm{H}$ & & 3.435100289123 & -0.696995844898 & 1.117199323537 \\
\hline $\mathrm{H}$ & & -0.871249727965 & 1.798630273437 & -0.535511134104 \\
\hline $\mathrm{H}$ & & -3.790528012593 & -0.724047838668 & -0.435948937196 \\
\hline $\mathrm{H}$ & & -3.456377848448 & 0.716582534192 & 0.688503438531 \\
\hline $\mathrm{H}$ & & -2.192802861763 & -0.972834324779 & 0.468273040730 \\
\hline
\end{tabular}




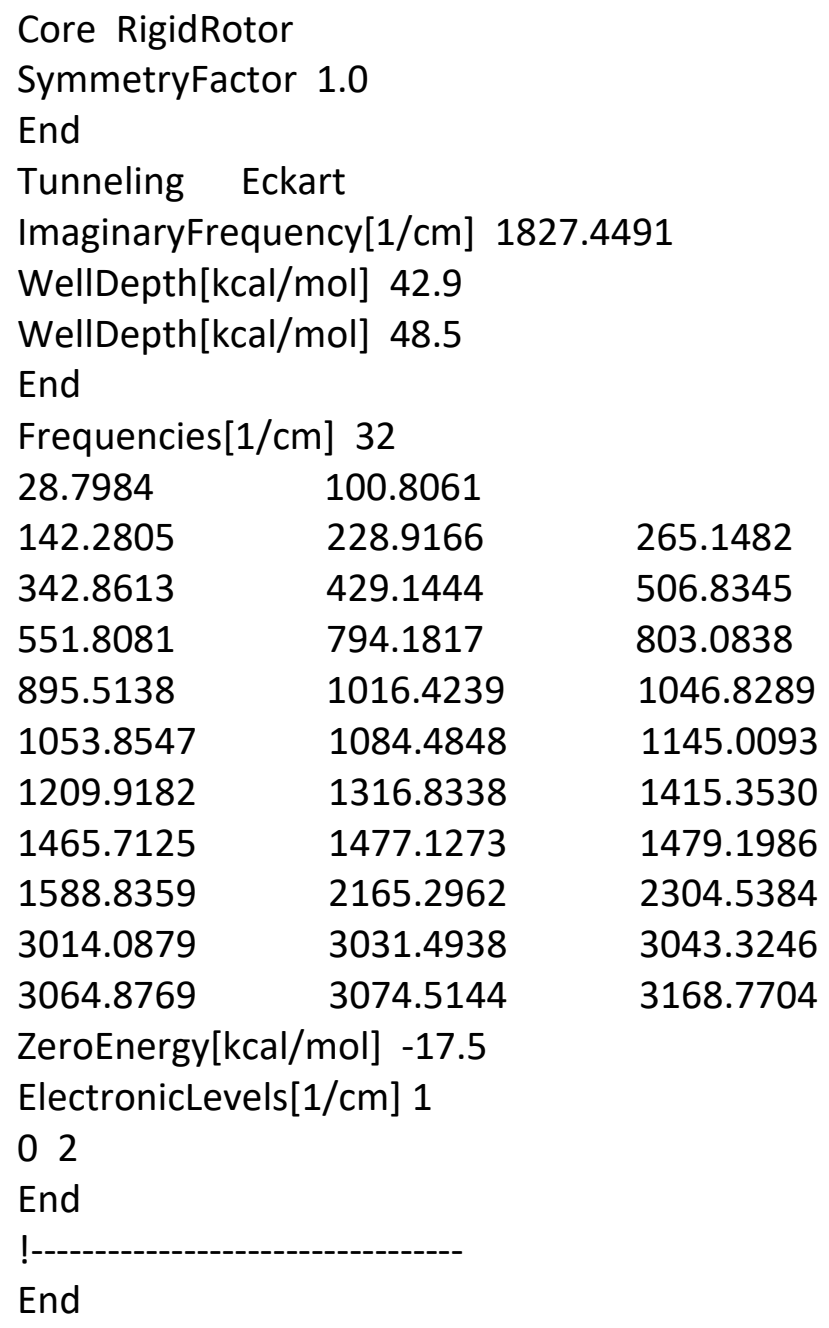

